\title{
LOCALLY PLANAR TOROIDAL GRAPHS ARE 5-COLORABLE
}

\author{
MICHAEL O. ALBERTSON ${ }^{1}$ AND WALTER R. STROMQUIST
}

\begin{abstract}
If a graph can be embedded in a torus in such a way that all noncontractible cycles have length at least 8 , then its vertices may be 5-colored. The conclusion remains true when some noncontractible cycles have length less than 8, if the exceptions are all homotopic. Essentially this hypothesis means that small neighborhoods of the graph are planar. No similar conclusion holds for 4-colorability.
\end{abstract}

Introduction. An $r$-coloring of a graph $G$ is an assignment of the colors $1,2, \ldots, r$ to the vertices of $G$ so that any two adjacent vertices are assigned different colors. A graph is said to be $r$-chromatic if it has an $r$-coloring but no $(r-1)$-coloring. It is well known that if $G$ is embedded on the torus then $G$ can be 7-colored [7]. Furthermore every 7-chromatic toroidal graph contains $K_{7}$ as a subgraph [5]. Such a characterization of $r$-chromatic toroidal graphs is beyond view for $3 \leqslant r \leqslant 5$ and likely to be difficult for $r=6$. Some possible characterizations for 6-chromatic toroidal graphs are suggested in the section of open questions at the end of this paper. One partial result in this direction is that there is a unique 6-chromatic toroidal graph which is regular of degree six [2]-a result which, like the theorem we present here, is based on the Four Color Theorem [3, 4].

Many of the attacks upon the Four Color Problem (including the eventually successful one of finding an unavoidable set of reducible configurations) have been based upon the fundamental premise that most obstructions to 4-coloring are "local" in nature. Thus if $G$ is a toroidal graph which locally appears to be planar it might be reasonable to believe that $G$ is 4-colorable. Surprisingly, this is not always true. Steve Fisk has constructed 5-chromatic graphs that can satisfy any notion of "local planarity" on any surface except the sphere [6]. As his result is ingenious yet simple, we include a proof of it following the proof of our main result.

In this paper we intend to prove that locally planar graphs on the torus are 5-colorable. The first step is to find a precise definition which corresponds to the vague notion of locally planar. Let $G$ be a graph embedded in a torus $T$. A cycle in $G$ is said to be minimal if it has no diagonals in $G$, and noncontractible, or $n c$ - if it is not homotopic to a point. Let $C_{0}$ be an nc-cycle of minimal length, and among

Received by the editors April 22, 1980 and, in revised form, April 22, 1981. Presented at the 83rd summer meeting of the AMS, August 25, 1979.

1980 Mathematics Subject Classification. Primary $05 C 15$.

Key words and phrases. Toroidal graph, vertex coloring.

${ }^{1}$ Research supported in part by NSF Grant MCS 77-03940.

() 1982 American Mathematical Society 0002-9939/81/0000-1042/\$03.00 
minimal nc-cycles not homotopic to $C_{0}$, let $C_{1}$ be of minimal length. Then $W(G)=\left|C_{0}\right|$ is called the width of $G$, and $W^{*}(G)=\left|C_{1}\right|$ is called the second width of $G$. These numbers are independent of the choice of $C_{0}$ and $C_{1}$, although in special cases they may depend on the way in which $G$ is embedded in $T$. This definition of $W^{*}(G)$ agrees with the definition in [2].

We assert (without proof) that the following conditions are equivalent on the torus: (a) the $k$ th neighborhood of every vertex in $G$ has a neighborhood in $T$ which is planar, and (b) $W^{*}(G)>2 k+2$. Thus lower bounds on $W^{*}$ are essentially requirements that $G$ be "locally planar".

The purpose of this paper is to prove the following.

THEOREM. If $G$ is embedded in the torus with $W^{*}(G)>8$, then $G$ can be 5-colored.

The proof of the theorem has three major parts. First we prove that without loss of generality we may assume that $G$ is "orderly". Second we prove that if $G$ is orderly then $G$ contains a "nice" nc-cycle. Finally we prove that if $C$ is this nice nc-cycle then there is a 4-coloring of $G-C$ which extends to a 5-coloring of $G$ provided $W^{*}$ is large enough. Some steps of the proof do not work when $W(G)=3$, so that case is treated separately at the end of the proof.

$G$ is orderly. A graph $G$ embedded on any surface is said to be orderly if

(1) $G$ is a triangulation,

(2) every contractible 3-cycle is a face boundary, and

(3) every contractible 4-cycle is either the first neighbor circuit of a vertex of degree four or the mod 2 sum of two face boundaries which share a common edge.

(We do not allow loops or multiple edges in any case.) Given an embedded graph $G$ we will transform $G$ into an orderly graph $G_{0}$ with the property that $W\left(G_{0}\right)=W(G)$ and $W^{*}\left(G_{0}\right)=W^{*}(G)$. First we triangulate the interior of every nontriangular face of $G$ as follows.

(a) Subdivide the face; i.e., insert a new vertex adjacent to every vertex on the boundary of the face; and

(b) subdivide every new edge added inside a $k$-sided face, $k>6$, by adding $[(k-2) / 4]$ new vertices along the edge.

Repeated application of steps (a) and (b) will eventually yield a triangulation. Each new nc-cycle which is introduced is at least as long as some preexisting cycle of the same homotopy class, so $W$ and $W^{*}$ are unchanged. Now in this triangulation we erase all vertices interior to any contractible 3-cycle, and in every contractible 4-cycle which is not two adjacent triangles we erase the interior vertices and subdivide the newly created quadilateral. The resulting graph is our orderly $G_{0}$.

We now claim that if $G_{0}$ can be 5-colored then so can $G$. As we have subdivided only new edges, every pair of vertices, neither of which is interior to a contractible 3 - or 4-cycle, are adjacent in $G_{0}$ provided they are adjacent in $G$. Thus we have a 5-coloring of $G$ except for the interior of contractible 3- or 4-cycles. As the interior and boundary of a contractible 3-cycle form a planar graph any coloring of the bounding 3-cycle can be extended to a 4-coloring of the interior. It is not true that 
every coloring of the boundary of a 4-cycle can be extended to a 4-coloring of the interior; but any coloring of the vertices of a 4-cycle can be extended to a 5-coloring of any planar graph interior to the 4-cycle (this is shown, for example, in Levow [8]), which is sufficient.

From now on we assume that $G$ is orderly.

$G$ contains a nice nc-cycle. An nc-cycle $C$ in an embedded graph is said to be nice if

(1) $C$ is minimal (no diagonals), and either

(2a) $|C|$ is even, or

(2b) $C$ contains a vertex of degree four.

Let $G$ be an embedded graph and let $C_{0}$ be an nc-cycle of minimal length; that is, $\left|C_{0}\right|=W(G)$. Let $L\left(C_{0}\right)$ and $R\left(C_{0}\right)$ be the "left" and "right" neighbor cycles of $C_{0}$ (see Figure 1). (In general $L\left(C_{0}\right)$ and $R\left(C_{0}\right)$ need not be minimal cycles, nor do they have to be disjoint.)

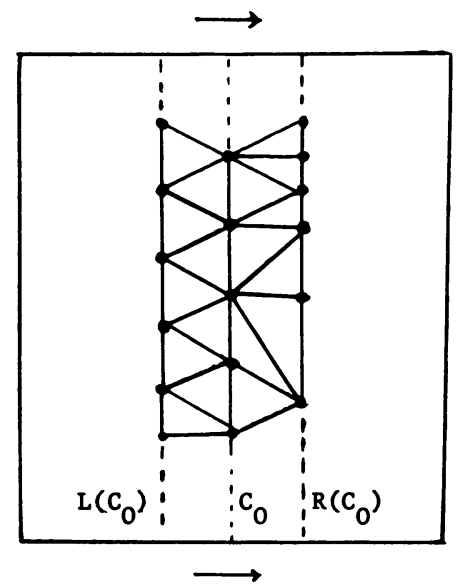

Figure 1

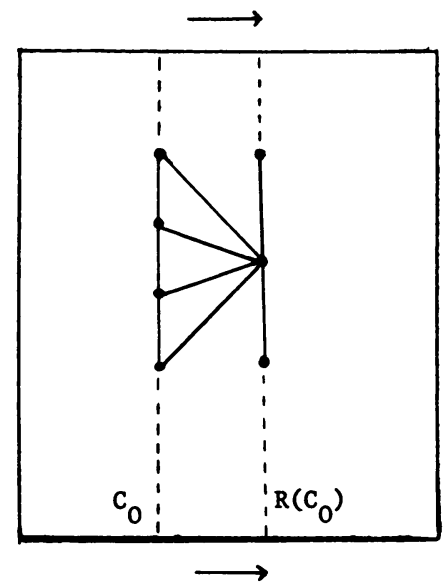

Figure 2 
In this section of the proof we will show that $G$ contains a nice nc-cycle whose vertices are contained in $C_{0}$ and $R\left(C_{0}\right)$. The case $\left|C_{0}\right|=3$ will be treated later. If $\left|C_{0}\right|=4$ (or any even number) there is nothing to prove. Hence we may assume that $\left|C_{0}\right| \geqslant 5$ and that $\left|C_{0}\right|$ is odd. If there were a vertex of $C_{0}$ with no neighbors in $R\left(C_{0}\right)$, then $C_{0}$ would not be minimal since $G$ is a triangulation. If there were two consecutive vertices of $C_{0}$ each of which had exactly one neighbor in $R\left(C_{0}\right)$, then $C_{0}$ would not be of minimal length among nc-cycles (Figure 2). Since $\left|C_{0}\right|$ is odd, it follows that $C_{0}$ contains two consecutive vertices each of which has at least two neighbors in $R\left(C_{0}\right)$. Call these vertices $X_{3}$ and $X_{4}$. They must have a unique common neighbor $Y$ in $R\left(C_{0}\right)$ which is not adjacent to any other vertex of $C_{0}$. Label other vertices $X_{1}, X_{2}, X_{5}, X_{6}$ as in Figure 3 and let $D$ consist of all vertices of $C_{0}$ other than $X_{1}, \ldots, X_{6}$. (It is possible that $X_{1}=X_{6}$ and $D$ is void.) Let $\Gamma_{1}$ denote a minimum-length path from $X_{1}$ to $Y$ every interior vertex of which is a neighbor of $X_{3}$ in $C_{0}$ or $R\left(C_{0}\right)$. (Note that $\Gamma_{1}$ may or may not include $X_{2}$.) Consider the cycle $\left(D X_{1} \Gamma_{1} Y X_{4} X_{5} X_{6}\right)$. It is straightforward to check that this cycle is minimal: any diagonals will produce an nc-cycle in $G$ with fewer than $\left|C_{0}\right|$ vertices. Clearly this cycle is homotopic to $C_{0}$. If $\left|\Gamma_{1}\right|$ is even, then this cycle is even and consequently nice; so we may assume that $\left|\Gamma_{1}\right|$ is odd. Let $\Gamma_{2}$ be a minimum-length path from $Y$ to $X_{6}$ every interior vertex of which is a neighbor of $X_{4}$ in $C_{0}$ or $R\left(C_{0}\right)$. As above either $\left(D X_{1} X_{2} X_{3} Y \Gamma_{2} X_{6}\right)$ is nice or $\left|\Gamma_{2}\right|$ is odd. In the latter case consider $\left(D X_{1} \Gamma_{1} Y \Gamma_{2} X_{6}\right)$; it is an nc-cycle of even length so either it is nice or it has a diagonal. The only diagonal which is consistent with our assumptions is an edge from a vertex of $\Gamma_{1}$ to a vertex of $\Gamma_{2}$. Such an edge produces a 4-cycle in which $Y$ is an interior vertex; therefore $Y$ has degree 4 and $\left(D X_{1} \Gamma_{1} Y X_{4} X_{5} X_{6}\right)$ is nice. Figure 4 shows that this behavior can in fact occur.

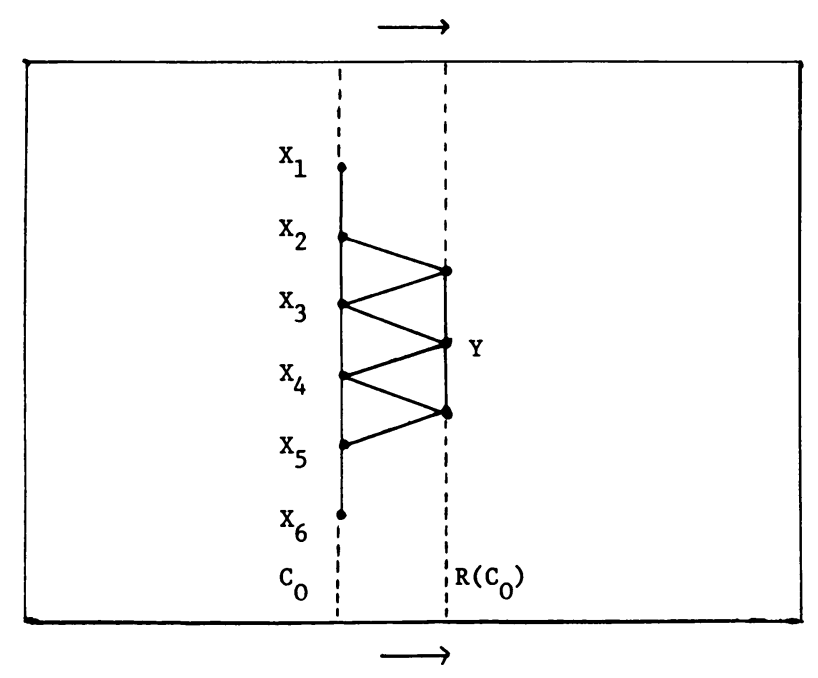

FIGURE 3 


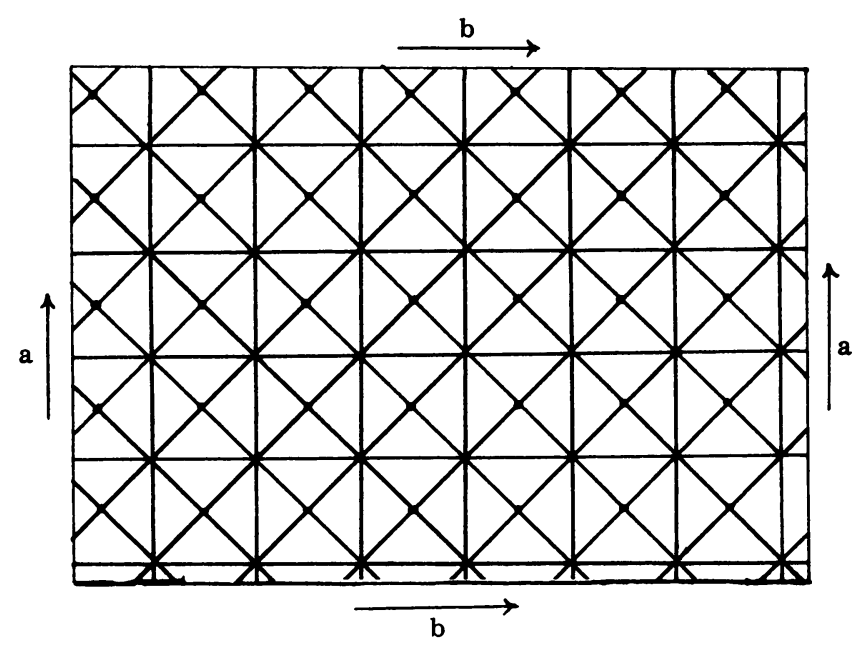

FIGURE 4. A toroidal graph in which every minimal circuit homotopic to $C_{0}$ or $C_{1}$ has odd length

Now assume that $W(G) \geqslant 4$ and $W^{*}(G) \geqslant 8$, let $\left|C_{0}\right|=W(G)$, and let $C$ be a nice nc-cycle contained in $C_{0} \cup R\left(C_{0}\right)$. If $W(G)$ is even we may take $C=C_{0}$. Suppose some vertex of $R(C)$ is connected to some vertex of $L(C)$. Then two vertices of $C_{0}$ are connected by a path of length four (three if $W(G)$ is even) which is not homotopic to any segment of $C_{0}$. Such a path combined with at most half of $C_{0}$ forms an nc-cycle, not homotopic to $C_{0}$, whose length is either less than $\left|C_{0}\right|$ or less than 8 . This being impossible, we may assume that there is no edge connecting any vertex of $L(C)$ to any vertex of $R(C)$.

Coloring. Let $C$ be the nice nc-cycle chosen above. We first produce a 4-coloring of $G-C$ which contains only three colors on $L(C)$ and only three colors on $R(C)$. Construct $G^{\prime}$ from $G-C$ by adding a vertex $X_{L}$ adjacent to every vertex of $L(C)$ and a vertex $X_{R}$ adjacent to every vertex of $R(C)$. If $G$ is toroidal then $G^{\prime}$ is planar and hence 4-colorable; and any 4-coloring of $G^{\prime}$ yields the desired coloring of $G-C$.

We will now extend this coloring of $G-C$ to a 5-coloring of $G$. If $L(C)$ contains the same three colors as $R(C)$, say $\{1,2,3\}$, then colors 4 and 5 may be assigned alternately to the vertices of $C$. This works if $|C|$ is even; if $|C|$ is odd it still works provided the vertex of degree four is saved until last when it can be assigned a color not used on its neighbors.

Suppose instead that $L(C)$ and $R(C)$ contain different sets of three colors: say $\{1,2,3\}$ on $L(C)$ and $\{1,2,4\}$ on $R(C)$. Then we may change each vertex on $L(C)$ with color 3 to 5 , and change each vertex on $R(C)$ with color 4 to 5 . This creates no conflicts since no edges connect $R(C)$ to $L(C)$. Now colors 3 and 4 are available for coloring the vertices of $C$, and the coloring may be completed as above. 
The case $W(G)=3$. Let $G$ contain an nc-cycle $C$ of length 3 , and assume that $W^{*}(G) \geqslant 7$. We will construct a 5-coloring of $G$. First slice the torus along $C$ to produce a planar graph $G^{\prime}$ containing two copies of the vertices of $C$, labelled $X_{1}$, $X_{2}, X_{3}, Y_{1}, Y_{2}, Y_{3}$ as in Figure 5. If a 5-coloring of $G^{\prime}$ can be found in which the color of $X_{i}$ matches the color of $Y_{i}$ for each $i=1,2,3$, it can be sewn together to give a 5-coloring of $G$.

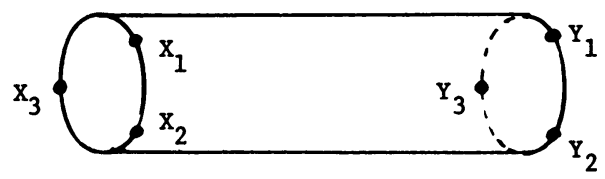

FIGURE 5

Being planar, $G^{\prime}$ has a coloring using only the colors $1,2,3,4$. If $X_{i}$ matches $Y_{i}$ for at least two subscripts, say $i=1$ and 2 , the coincidence can be completed by changing the colors of both $X_{3}$ and $Y_{3}$ to 5 . Otherwise there must be one color which occurs on both copies of $C$ but not on corresponding vertices. Without losing generality we may assume that both $X_{1}$ and $Y_{2}$ have color 1 . Let $a$ be the color of $X_{2}$ and $b$ the color of $Y_{1}$, as in Figure 6. Of course $a$ and $b$ need not be distinct.

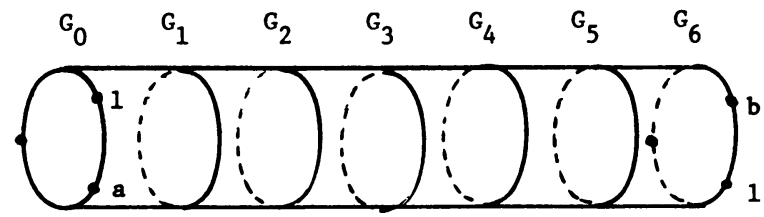

Figure 6

Now let $G_{0}=\left\{X_{1}, X_{2}, X_{3}\right\}$. For $j=1, \ldots, 5$, let $G_{j}$ be the set of vertices of $G^{\prime}$ which can be reached by paths of length $j$ from $G_{0}$, but by no shorter paths. Let $G_{6}$ consist of all vertices of $G^{\prime}$ not in $G_{0}, \ldots, G_{5}$. The condition $W^{*}(G)>7$ implies that $Y_{1}, Y_{2}, Y_{3}$ are in $G_{6}$. There is no edge joining $G_{j}$ to $G_{k}$ if $j, k$ differ by more than 1. Now alter the coloring of $G^{\prime}$ as follows:

On $G_{0}$, exchange colors 1 and $a$, and change the color of $X_{3}$ to 5 .

On $G_{1}$, exchange colors 1 and $a$.

On $G_{2}$, change all colors $a$ to 5 , and all colors 1 to $a$.

On $G_{3}$, change all colors $a$ to 5 .

On $G_{4}$, change all colors $a$ to 5 , and all colors $b$ to $a$.

On $G_{5}$, exchange colors $b$ and $a$.

On $G_{6}$, exchange colors $b$ and $a$, and change the color of $Y_{3}$ to 5 .

No conflicts occur, and $X_{i}$ is made to match $Y_{i}$ for each $i$. Thus the required 5-coloring of $G$ is completed.

Fisk's examples. The construction of 5-chromatic graphs with arbitrarily large widths relies on the following lemma.

LEMMA. Suppose $G$ is a triangulation of any surface with exactly two vertices of odd degree. A 4-coloring of $G$ must assign the two vertices of odd degree the same color. 
Proof. Fix a 4-coloring. An edge (face) of $G$ will be called an $i-j$ edge $(i-j-k$ face) provided its incident vertices are colored $i$ and $j(i, j$ and $k)$. Suppose the number of $1-2-3$ faces is congruent to $q(\bmod 2)$. As each $1-2-k$ face is incident with a unique 1-2 edge and each 1-2 edge is incident with exactly two such faces, the number of faces incident with a vertex colored 1 and a vertex colored 2 is even. Thus the number of 1-2-4 faces (and by an obvious extension the number of $i-j-k$ faces) is also congruent to $q(\bmod 2)$. For any $i$, the total number of faces incident with all of the vertices colored $i$ is congruent to $3 q(\bmod 2)$ and hence congruent to $q(\bmod 2)$. This number is also the sum of the degrees of all vertices colored $i$. As there are only two vertices of odd degree, $q$ must be 0 and both vertices of odd degree are assigned the same color. This proves the lemma.

Evidently if a triangulation has exactly two vertices of odd degree which are adjacent, then it cannot be 4-colored. Examples of such triangulations with arbitrarily large widths can be found on any surface except the sphere. Figure 7 exhibits one such triangulation of the torus.

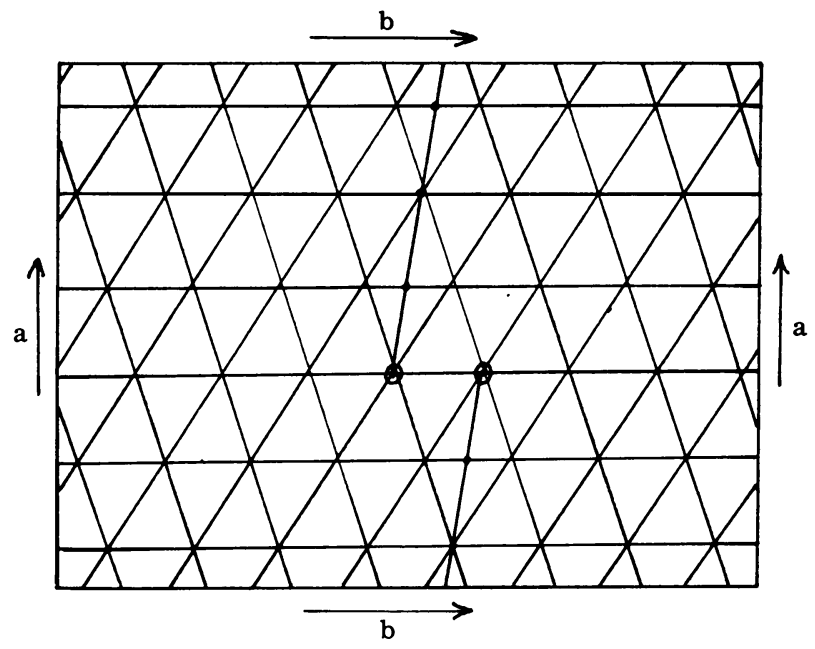

FIGURE 7. A locally-planar toroidal graph with only two odd vertices, which are adjacent

\section{Open questions.}

1. Does there exist a 6-chromatic toroidal graph with $3<W(G)<8$ ?

2. What are the vertex critical 6-chromatic toroidal graphs? Currently five such graphs are known.

3. Is there a finite number of vertex critical 6-chromatic toroidal graphs?

4. Does there exist a locally planar 5-chromatic triangulation of the torus which does not contain a "Fisk graph" [10]?

5. Is there a constant $M$ such that all but $M$ of the vertices of any toroidal graph can be 4-colored? If $v$ denotes the number of vertices it is known that $G$ contains an nc-cycle with no more than $\sqrt{2 v}$ vertices [1]. Thus all but $\sqrt{2 v}$ of the vertices of a toroidal graph can be 4-colored. 
6. Conjecture. Every 6-chromatic graph on the torus which is not regular of degree six contains $K_{5}$ (H. J. Straight [9]).

We suspect that locally planar graphs on any surface are 5-colorable, given a suitable definition of local planarity. If width is defined for other surfaces in the same way as for the torus, we conjecture that embedded graphs whose width exceeds some bound must be 5-colorable. Such a bound would necessarily depend on the genus and the orientability of the surface.

ADDED IN PROOF. Phil Huneke informs us that Fisk's lemma in the context of maps on the sphere appeared in J. P. Ballantine, A postulational introduction to the four color problem, Six Studies in Mathematics, Univ. of Washington Publ. Math., Vol. 2, No. 1, pp. 1-15.

\section{REFERENCES}

1. M. O. Albertson and J. P. Hutchinson, On the independence ratio of a graph, J. Graph Theory 2 (1978), 1-8.

2. __ On six-chromatic toroidal graphs, Proc. London Math. Soc. (3) 41 (1980), 533-556.

3. K. Appel and W. Haken, Every planar map is four colorable. Part I: Discharging, Illinois J. Math. 21 (1977), 429-490.

4. K. Appel, W. Haken and J. Koch, Every planar map is four colorable. Part II: Reducibility, Illinois J. Math. 21 (1977), 491-567.

5. G. A. Dirac, Short proof of a map colour theorem, Canad. J. Math. 9 (1957), 225-226.

6. S. Fisk, The non-existence of colorings, J. Combin. Theory Ser. B 24 (1978), 247-248.

7. P. J. Heawood, Map-colour theorem, Quart J. Pure Appl. Math. 24 (1890), 332-338.

8. R. B. Levow, Coloring planar graphs with five or more colors, Proc. 5th S.-E. Conf. Combinatorics, Graph Theory, and Computing, Utilitas Math. Publ., Winnepeg, 1974, pp. 549-561.

9. H. J. Straight, Note on the cochromatic number of several surfaces, J. Graph Theory 4 (1980), 115-117.

10. W. Stromquist, The four color problem for locally planar graphs, Graph Theory and Related Topics, Academic Press, New York, 1979, pp. 369-370.

Department of Mathematics, Smith College, Northampton, Massachusetts 01063

Daniel H. Wagner, Associates, Paoli, Pennsylvania 19301 